\title{
Heavy Metal Incorporation During Goethite Oriented Attachment
}

\author{
S. T. MergelsberG ${ }^{1 *}$, G. ZhU ${ }^{1}$, J. A. Soltis ${ }^{1}$, M. E. \\ MCBRIARTY ${ }^{1}$, E. NAKOUZi ${ }^{1}$, J. J. DE YOREO ${ }^{1}$, \\ E. S. ILTON ${ }^{1}$ \\ ${ }^{1}$ Pacific Northwest National Laboratory, Richland, WA \\ 99352, USA (*correspondence: \\ sebastian.mergelsberg@pnnl.gov)
}

Nucleation and growth of iron (oxyhydr)oxides in natural environments often occur in mixed cation systems, where minor and trace elements co-precipitate with abundant phases. Less well understood is whether these elements can be incorporated into host minerals by non-classical growth pathways. For example, goethite can grow by oriented attachment (OA) of co-aligned precursor nanoparticles. As a result, the composition of the mineral is a function of both 1) element compatibility and 2) growth mechanism. To discern between these two competing controls on incorporation, this study focuses on the incorporation of $\mathrm{U}(\mathrm{VI})$ and $\mathrm{W}(\mathrm{VI})$ during the growth of goethite by OA.

Measurements of U(VI) incorporation into goethite by ICPOES, XRD, and TEM showed that U(VI) additions early in the OA process hindered crystallization [1]. However, the amount of sorbed $\mathrm{U}(\mathrm{VI})$ was highest when added early during $\mathrm{OA}$ and decreased over time, eventually converging to similar values for both early and late additions of U(VI). Evidence from scanning transmission electron microscopy (STEM) indicated a portion of the acid resistant sorbed $\mathrm{U}(\mathrm{VI})$ was likely associated with grain boundaries between aggregating particles, not nanopores. These features - and the associated $\mathrm{U}(\mathrm{VI})$ - were annealed out with increasing reaction time. Further evidence that $\mathrm{U}(\mathrm{VI})$ was not incorporated into the structure of goethite was acquired by time elapsed imaging of $U$ atom diffusion and extended X-ray absorption fine structure spectra (EXAFS). Quantification by $a b$ initio molecular dynamics informed EXAFS showed an upper solubility limit of only U/Fe $\sim 0.02$ atom $\%$ in goethite where $\mathrm{U}(\mathrm{VI})$ was added early in the process.

To determine the effect of heavy metal coordination chemistry, we are comparing and contrasting the incorporation of W(VI) and $\mathrm{U}(\mathrm{VI})$ into goethite during OA under identical conditions. Unlike uranyl(VI), tungsten(VI) does not have unreactive uranyl oxygens that were shown to impede $\mathrm{U}(\mathrm{VI})$ incorporation into goethite during OA. Further, addition of U(VI) or W(VI) during the formation of ferrihydrite - the metastable OA precursor - may further constrain the solubility of these elements in the final goethite structure.

[1] Soltis, J.A., et al. (2019) Environmental Science: Nano 6(10), 3000-3009. 